\title{
Survival of patients with schistosomiasis- associated pulmonary arterial hypertension in the modern management era
}

\author{
To the Editor:
}

Pulmonary arterial hypertension (PAH) is a life-threatening disease characterised by an elevated pulmonary vascular resistance in the absence of left ventricular disease, and increasing pulmonary artery pressure, with consequent right ventricular failure and death [1]. Several aetiologies have been associated with $\mathrm{PAH}$, including connective tissue diseases, congenital heart diseases and chronic infections, such as HIV. Due to its high prevalence in developing countries, one of the most relevant forms of PAH worldwide is the one associated to schistosomiasis [2].

Schistosomiasis-associated pulmonary arterial hypertension (Sch-PAH) is present in $5 \%$ of patients with the hepatosplenic form of the disease [3]. Due to its histological and haemodynamic similarities to idiopathic PAH (IPAH), it is classified within group 1 (pre-capillary pulmonary hypertension) of the current pulmonary hypertension classification [4]. However, despite the similarities, a previous cohort has shown that Sch-PAH has a distinct, more benign course than IPAH, even in the absence of specific therapy [5].

Data regarding the efficacy of specific PAH therapy in Sch-PAH are scarce. Improvements in functional class, cardiac output and 6-min walking test distance (6MWT), using phosphodiesterase-5 inhibitors (PDE5i) or endothelin-1 receptor antagonists (ERA) were demonstrated in a small cohort of 12 Sch-PAH patients [6]. The effect of PAH treatment on hard end-points, such as clinical worsening or survival, has not been evaluated in this population, until this date.

The aim of this study was to compare the survival of newly diagnosed Sch-PAH patients treated with PAH targeted therapies against a group of untreated patients from a historical cohort.

Data from all consecutive, newly diagnosed Sch-PAH patients referred to our centre in Brazil were analysed. Sch-PAH was characterised by the presence of mean pulmonary arterial pressure $\geqslant 25 \mathrm{mmHg}$ with pulmonary artery occlusion pressure $\leqslant 15 \mathrm{mmHg}$, in the absence of significant lung parenchymal disease, left ventricular dysfunction or chronic thromboembolic disease, associated with the presence of periportal fibrosis and/or left liver lobe enlargement associated with at least one of the following features: positive epidemiology, previous treatment of schistosomiasis or identification of eggs in stool or rectal biopsy [7]. Since specific PAH therapy became widely available in Brazil after 2010, patients diagnosed before this date received only supportive therapy and comprised our historical cohort. After 2010, Sch-PAH patients were regularly treated with PDE5i, ERA or both, at discretion of the attending physician, in accordance to the available guidelines $[8,9]$.

Baseline clinical, demographical, including New York Heart Association (NYHA) functional class (FC) assessment, 6MWT, brain natriuretic peptide (BNP) and haemodynamic data were collected.

Analysis was performed using the SPSS 21 statistical package (SPSS, Inc.). All continuous variables are expressed as mean $\pm \mathrm{SD}$ and compared using $\mathrm{t}$-test. Categorical data are presented as proportions and compared using Chi-square or Fisher's exact test, as appropriate.

The 60-month survival was evaluated using Kaplan-Meier estimate. Log-rank test was used for curve comparison. A p-value $<0.05$ was considered statistically significant.

@ERSpublications

Survival in schistosomiasis associated pulmonary arterial hypertension has improved in recent years with the use of targeted therapies http://ow.ly/perr30jrhBo

Cite this article as: Fernandes CJC, Piloto B, Castro M, et al. Survival of patients with schistosomiasisassociated pulmonary arterial hypertension in the modern management era. Eur Respir J 2018; 51: 1800307 [https://doi.org/10.1183/13993003.00307-2018]. 
The study population comprised 102 Sch-PAH patients; 50 patients (49\%) were diagnosed before 2010 and, therefore, did not receive specific PAH therapy (untreated group; historical control). Except for the availability of specific treatment, there was no change in the standard of care during the whole observation period. 52 patients (51\%) were diagnosed after 2010 and received targeted therapies: 41 patients $(40.2 \%)$ received monotherapy with PDE5i, 10 patients $(9.8 \%)$ received monotherapy with ERA, and one patient (1\%) received upfront combination therapy.

Sch-PAH untreated and treated patients had similar baseline clinical and demographic data. At diagnosis, the mean \pm SD age was $53.7 \pm 13.2$ versus $52.7 \pm 13.0$ years, $6 \mathrm{MWT}$ was $458 \pm 149 \mathrm{~m}$ versus $411 \pm 100 \mathrm{~m}$, and BNP was $107 \pm 90$ versus $142 \pm 183 \mathrm{pg} \cdot \mathrm{dL}^{-1}$. Among untreated patients, $68 \%$ were female, with 25 patients (55\%) in FC I and II and 20 patients (45\%) in FC III and IV, whilst in the treated group, 59.6\% were female patients, with 26 patients (51\%) in FC I and II and 25 patients (49\%) in FC III and IV ( $p>0.05$ for all comparisons).

The haemodynamic impairment was also similar between groups. Untreated and treated patients presented right atrium pressure of $9.7 \pm 4.7$ and $10.8 \pm 5.7 \mathrm{mmHg}(\mathrm{p}=0.281)$; mean pulmonary arterial pressure of $52.4 \pm 16.7$ versus $59.9 \pm 17.9 \mathrm{mmHg}(\mathrm{p}=0.033)$, pulmonary artery occlusion pressure of $11.7 \pm 4.1$ versus $13.4 \pm 5.4 \mathrm{mmHg}(\mathrm{p}=0.085)$, cardiac output of $4.8 \pm 1.6$ versus $4.4 \pm 1.4 \mathrm{~L} \cdot \mathrm{min}^{-1}(\mathrm{p}=0.208)$ and pulmonary vascular resistance of $10.1 \pm 7.1$ versus $11.8 \pm 6.4 \mathrm{WU}(\mathrm{p}=0.225)$, respectively. No statistically significant difference was found between the groups. None of the patients presented acute vasodilator response, regardless of the group [10].

The survival rate of the untreated group at 60 months was $69.2 \%$ for the untreated and $89.1 \%$ for the treated Sch-PAH patients $(\mathrm{p}=0.029)$ (figure 1).

The use of specific PAH therapy in Sch-PAH patients was associated with improved survival, in comparison with a historical control group of untreated patients. To our knowledge, this is the first time that this information is reported in medical literature.

Schistosomiasis is the third most prevalent form of chronic infection disease worldwide, possibly infecting 200 million people [11]. Considering the prevalence of 5\% of PAH in patients with the hepatosplenic form of the disease [3], Sch-PAH is clearly one of the most relevant forms of PAH, particularly in developing countries $[12,13]$. Nevertheless, this population is rarely included in the large trials evaluating PAH targeted therapies [14]. Despite that, current $\mathrm{PAH}$ guideline recommends specific treatment for schistosomiasis, in line with other entities included in group 1 of the current PH classification [9]. However, the long-term efficacy of PAH targeted therapies had never been demonstrated in Sch-PAH.

Our study has some limitations that need to be highlighted. It is a non-randomised single-centre study, using historical controls as comparators, thus limiting the availability of follow-up data regarding the effect of targeted therapies in the functional and haemodynamic variables. However, our centre is the largest referral centre in the country, receiving patients from all other regions, including those from highly endemic areas. Furthermore, survival of untreated Sch-PAH survival rate was consistent with previously published data $[5,15]$. Likewise, the better survival associated with treatment is in line with the clinical and haemodynamic benefits of targeted PAH therapy in Sch-PAH previously demonstrated [6].

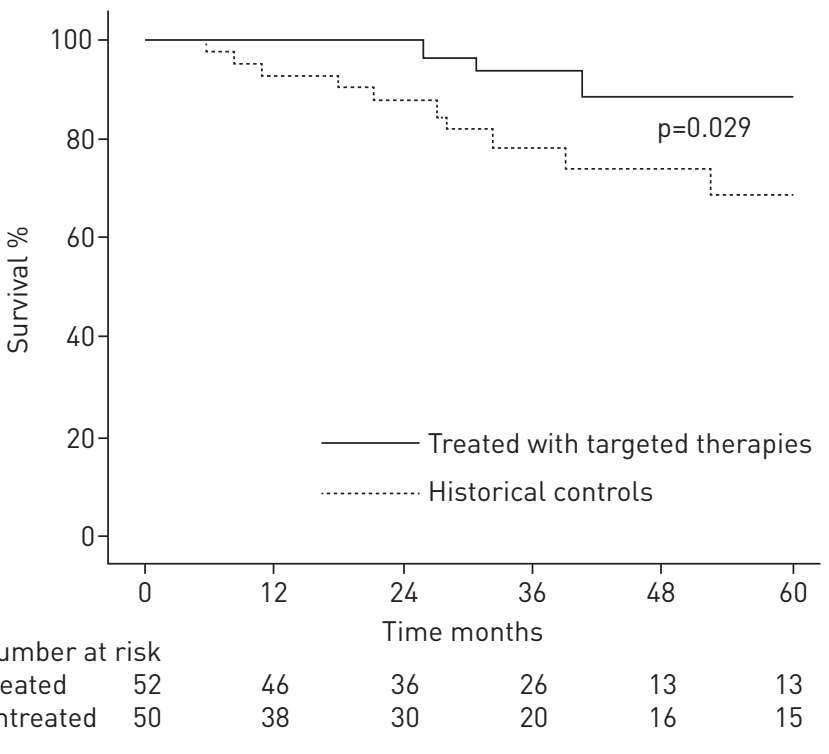

FIGURE 1 Survival of patients with schistosomiasis-associated pulmonary arterial hypertension according to treatment.

\section{Treated 52}

Untreated 50
46
38 
In conclusion, our results provide more robust evidence supporting current $\mathrm{PAH}$ guidelines recommendation, reinforcing the indication for targeted therapies in Sch-PAH, similarly to other forms of PAH.

Caio Julio Cesar Fernandes $\bullet$, Bruna Piloto, Marcela Castro, Francisca Gavilanes Oleas, Jose Leonidas Alves Jr, Luis Felipe Lopes Prada, Carlos Jardim and Rogerio Souza $\mathbb{\bullet}$

Pulmonary Division, Heart Institute, University of Sao Paulo Medical School, Sao Paulo, Brazil.

Correspondence: Rogerio Souza, Pulmonary Dept, Heart Institute, University of Sao Paulo Medical School, Av. Dr. Eneas de Carvalho Aguiar, 44, Sao Paulo, Brazil 05403-000. E-mail: souza.rogerio@me.com

Received: Feb 102018 | Accepted after revision: March 252018

Conflict of interest: C.J.C. Fernandes reports personal fees from Actelion Pharmaceuticals and Bayer, outside the submitted work. C. Jardim reports personal fees from GSK, Brentford, UK (employee, global medical expert). R. Souza reports lecture and consultancy fees from Actelion, Bayer, GSK and Pfizer.

\section{References}

Rubin LJ. Primary pulmonary hypertension. N Engl J Med 1997; 336: 111-117.

2 Fernandes CJ, Jardim CV, Hovnanian A, et al. Schistosomiasis and pulmonary hypertension. Expert Rev Respir Med 2011; 5: 675-681.

3 Lapa M, Dias B, Jardim C, et al. Cardiopulmonary manifestations of hepatosplenic schistosomiasis. Circulation 2009; 119: 1518-1523.

4 Simonneau G, Gatzoulis MA, Adatia I, et al. Updated clinical classification of pulmonary hypertension. J Am Coll Cardiol 2013; 62: D34-D41.

5 dos Santos Fernandes CJ, Jardim CV, Hovnanian A, et al. Survival in schistosomiasis-associated pulmonary arterial hypertension. J Am Coll Cardiol 2010; 56: 715-720.

6 Fernandes CJ, Dias BA, Jardim CV, et al. The role of target therapies in schistosomiasis-associated pulmonary arterial hypertension. Chest 2012; 141: 923-928.

7 Hovnanian A, Hoette S, Fernandes CJ, et al. Schistosomiasis associated pulmonary hypertension. Int J Clin Pract Suppl 2010; 64:Suppl .165, 25-28.

8 Galie N, Hoeper MM, Humbert M, et al. Guidelines for the diagnosis and treatment of pulmonary hypertension: the task force for the diagnosis and treatment of pulmonary hypertension of the European Society of Cardiology (ESC) and the European Respiratory Society (ERS), endorsed by the International Society of Heart and Lung Transplantation (ISHLT). Eur Heart J 2009; 30: 2493-2537.

9 Galie N, Humbert M, Vachiery JL, et al. 2015 ESC/ERS guidelines for the diagnosis and treatment of pulmonary hypertension: the joint task force for the diagnosis and treatment of pulmonary hypertension of the European Society of Cardiology (ESC) and the European Respiratory Society (ERS). Eur Respir J 2015; 46: 903-975.

10 Costa EL, Jardim C, Bogossian HB, et al. Acute vasodilator test in pulmonary arterial hypertension: Evaluation of two response criteria. Vascul Pharmacol 2005; 43: 143-147.

11 Souza R, Fernandes CJ, Jardim CV. Other causes of PAH (schistosomiasis, porto-pulmonary hypertension and hemolysis-associated pulmonary hypertension). Semin Respir Crit Care Med 2009; 30: 448-457.

12 Gavilanes F, Fernandes CJ, Souza R. Pulmonary arterial hypertension in schistosomiasis. Curr Opin Pulm Med 2016; 22: 408-414.

13 Hoeper MM, Humbert M, Souza R, et al. A global view of pulmonary hypertension. Lancet Respir Med 2016; 4: 306-322.

14 Papamatheakis DG, Mocumbi AO, Kim NH, et al. Schistosomiasis-associated pulmonary hypertension. Pulm Circ 2014; 4: 596-611.

15 Alves JL Jr, Gavilanes F, Jardim C, et al. Pulmonary arterial hypertension in the southern hemisphere: results from a registry of incident brazilian cases. Chest 2015; 147: 495-501. 